\title{
IMPACTOS DE LA NATURBANIZACIÓN EN LOS SERVICIOS AMBIENTALES
}

\author{
Maria-José Prados \& Júlia Maria Lourenço
}

\section{RESUMO}

El concepto de naturbanización fue definido recientemente para hacer referencia a la llegada de población y actividades urbanas a áreas de alto valor natural, tales como los Parques Nacionales (PPNN), generando cambios en la base socioeconómica y ecológica de las áreas de influencia y en el propio espacio protegido. Esta comunicación analiza la influencia de los procesos de naturbanización en los cambios de uso del suelo que parecen estar contribuyendo a la degradación de PPNN en España y Portugal. Ello lleva a la necesidad de controlar el impacto de la expansión urbana y de los procesos agrícolas intensivos sobre el medio natural. Al enfoque clásico de los espacios agrarios tradicionales como garantes del desarrollo territorial equilibrado, se incorporan por tanto otros como su papel en la conformación de paisajes culturales y sostén de elementos del patrimonio territorial, el mantenimiento de hábitats naturales, y el suministro de bienes y servicios ambientales.

\section{INTRODUCCION}

Las áreas protegidas juegan un papel central en la conservación de la biodiversidad. Sin embargo, éstas están expuestas a crecientes presiones, tales como la expansión urbana y el cambio climático. Este nuevo concepto de naturbanización explica cómo la presencia de un espacio natural protegido estimula los procesos de urbanización en las áreas de influencia. El reconocimiento de la calidad ambiental y paisajística de determinados ámbitos está motivando cambios en las preferencias residenciales de la población, que se muestran cada vez menos dependientes de las dinámicas impuestas por las áreas urbanas. La pérdida de atractivo de las ciudades como núcleos residenciales y productivos supone una nueva lógica en las motivaciones de los desplazamientos de la población. Los municipios localizados en las áreas de influencia de los parques nacionales presentan como rasgo diferencial un fuerte atractivo para la población que desea vivir, trabajar y disfrutar de su tiempo libre en las proximidades de espacios con una calidad ambiental demostrada.

El interés por estas áreas rurales remotas como nuevos enclaves residenciales se enmarca dentro del proceso de reestructuración rural y está repercutiendo en la dinámica urbanística y socioeconómica del territorio. La naturbanización no implica únicamente nuevas edificaciones para uso residencial (real o especulativo), infraestructuras para el acercamiento de estos espacios, o la provisión de los servicios y equipamientos para nuevos residentes. Además, identifica y valora el surgimiento de nuevas actividades productivas orientadas al "consumo de la naturaleza", como actividades de recreo y ocio, junto con la reactivación de otras relacionadas con el sector agropecuario. La planificación integrada del desarrollo urbano y la creación de nuevas infraestructuras, equipamientos y 
servicios; el seguimiento de la dinámica económica en el contexto de reestructuración rural unido junto al factor añadido de la existencia de un parque nacional; y la vinculación de la población residente con los objetivos de conservación ambiental son los ejes directrices que marcan la importancia del proceso de naturbanización.

Estas presiones producen potencialmente la degradación de los ecosistemas y los paisajes culturales, poniendo en riesgo la capacidad de los PPNN de acometer su función. La conservación de la biodiversidad tiene un papel clave en la preservación del medio natural y los paisajes culturales asociados a estos espacios protegidos. Asimismo garantizan la provisión de servicios ecosistémicos fundamentales para el desarrollo sostenible del territorio, tales como la producción de alimentos y materias primas, el suministro de agua y aire limpios, el control de inundaciones, la productividad del suelo y el funcionamiento del ciclo de los nutrientes, la prevención de procesos erosivos, etc. Si bien el mantenimiento de tales servicios requiere un mínimo de biodiversidad, una mayor riqueza aumenta la resiliencia de los ecosistemas y por tanto su capacidad de hacer frente a presiones.

Esta comunicación investiga los procesos de naturbanización en las áreas de influencia de los parques nacionales de Andalucía (Sur de España) y Nordeste de Portugal por tres razones principales. En primer lugar, ayuda a obtener una mejor comprensión de los aspectos que influyen en los procesos socio-demográficos de la población residente en las áreas de influencia, en especial nuevos residentes y diferencias con respecto a la población original. En segundo lugar, permite identificar el valor económico de los parques naturales en calidad de promotores de actividades económicas nuevas y complementarias de las tradicionales, y debido a las inversiones públicas en materia ambiental. Y en tercer lugar, hace posible disponer de los instrumentos para el seguimiento de cambios en la ocupación y usos del suelo por sus efectos sobre los paisajes. En definitiva, apoya los mecanismos necesarios para un mejor entendimiento de los objetivos de conservación, la importancia de la cohesión territorial y la preservación de las cualidades paisajísticas y medioambientales en los parques nacionales. Todos ellos suponen valores esenciales para lograr un buen entendimiento entre la población y el medio ambiente.

\section{PLANTEAMIENTO TEÓRICO}

El término naturbanización es una paráfrasis del de counterurbanization (contraurbanización en español) desarrollado a partir de la década de 1970 en los estudios de autores como Berry (1976 y 1978), Fielding (1986) y Champion (1989). En síntesis, éstos señalan un cambio en el comportamiento de los procesos de urbanización de países como Estados Unidos o Gran Bretaña, y que en palabras del propio Berry consistiría en "un proceso de desconcentración de población; (...) implica un movimiento de un estado de más concentración hacia un estado de menos concentración" (Champion, 1989: 20). A partir del comportamiento de la tasa de crecimiento demográfico anual entre 1950 y 1980 , calculada para ámbitos territoriales de diferente tamaño, sus investigaciones constatan la ralentización y reducción del tamaño de los considerados metropolitanos, a la par que las áreas menos urbanizadas o incluso áreas rurales, alejadas de los - hasta entonces - grandes focos de atracción demográfica, crecían en población.

Mientras que la mayor parte de los trabajos sobre movimientos de población y reestructuración de las áreas rurales analizan el hecho en dependencia del comportamiento de las áreas urbanas, la hipótesis de la "naturbanización" valora la importancia del 
reconocimiento ambiental como justificación de las nuevas pautas en la movilidad y preferencias residenciales de la población (Brown \& Wardel, 1980; Williams \& Jobes, 1990; Camarero, 1993; Feria, 1998). Es en este contexto en el que cobra importancia la caracterización sociodemográfica de las áreas naturbanizadas, intentando identificar la existencia de procesos de movilidad y el perfil de los nuevos residentes en relación con la conservación ambiental y paisajística (Elbersen \& Huigen, 1998; Elbersen \& Prados, 1999; Prados, 2005; Elbersen, 2005). El otro eje sobre el que se sustenta la hipótesis guarda relación con las actividades económicas que están siendo reactivadas o las nuevas que surgen utilizando la presencia del parque natural (Cózar, 2000; García-Bellido \& García, 2002). La naturbanización contribuye a identificar el valor mercantil de los parques, en relación tanto a las actividades económicas que se apoyan en su existencia como a la inversión pública en materia medioambiental.

Hasta la fecha, la hipótesis sobre la existencia de los procesos de naturbanización ha sido testada en relación con los cambios demográficos en las áreas de influencia de parques nacionales europeos, y también en el conjunto de la red de parques nacionales en España (véase Prados, 2000; 2002; 2005; 2009). Los resultados iniciales de las investigaciones llevadas a cabo confirman la existencia de estos cambios demográficos, y constatan la importancia de la calidad del entorno residencial, la accesibilidad y la posibilidad de desarrollar actividades económicas como factores de atracción de población hacia estos espacios. Asimismo, muestran con claridad las diferencias existentes entre los parques litorales, más influidos por la dinámica del turismo, la construcción o la nueva agricultura y la actividad industrial asociada. Los parques de interior también presentan rasgos de naturbanización, en algunos casos muy intensos debido a la ventaja comparativa de los territorios, en otros simplemente por la intensificación de la presión agraria o la proximidad a las principales áreas urbanas de la región (Tulla, 1993; Esteban, 2003). Pero en ambos casos parecen determinantes las repercusiones sobre las actividades tradicionales y la explotación de nuevos recursos.

El desbordamiento de las zonas urbanas se acompaña de la invasión de áreas agrícolas y de alto valor natural, siendo de fuerte intensidad en casos muy señalados (Delgado, 2008; Fontaine \& Rounsewell, 2009; Hall, 2008; Mcdonald, 2009). Ello lleva a la necesidad de planificar en la dirección de acotar y controlar el impacto de la expansión urbana y de los procesos agrícolas intensivos sobre el medio rural y natural. Entre ambos extremos es preciso reforzar los aspectos positivos: la atracción de población, el desarrollo de actividades económicas sostenibles y la puesta en valor de la agricultura tradicional. Analizar los servicios ambientales de los procesos de naturbanización, lleva implícito la defensa de aquellos modelos productivos que contribuyan a la preservación del medio y la conservación de los paisajes y el patrimonio territorial. Al enfoque clásico de los espacios agrarios tradicionales como garantes del desarrollo territorial equilibrado, se incorporan por tanto otros como su papel en la conformación de paisajes culturales y sostén de elementos del patrimonio territorial, el mantenimiento de hábitats naturales, y el suministro de bienes y servicios ambientales (Bills \& Gross, 2005; Fleskens, Duarte \& Eicher, 2009; Gómez, 2007; Swift, 2004). Uno de los mayores impactos derivado de estos procesos es la fragmentación de los ecosistemas producida por las modificaciones de los usos del suelo y sus impactos en la biodiversidad y los servicios ecológicos. Los ecosistemas naturales y humanos providencian un conjunto de servicios de importancia notable para la humanidad (Swift et al., 2004). De la agua, comida y materias primas hasta servicios no comerciales como la regulación climática, el procesamiento de la basura, la purificación del agua, el secuestro del carbono, el control de la erosión y de las inundaciones (Gómez-Bagetthun, de Groot, 2009). Veamos cuál es su comportamiento en ambos frentes. 
Los usos del suelo son la expresión territorial del aprovechamiento y la explotación de los recursos. El modo de aprovechamiento, entendido como explotación económica del medio, es un elemento central en la construcción y alteración de los paisajes culturales. La intensificación o la marginalidad de los modos de aprovechamiento conducen a cambios en las coberturas y usos de los suelos y éstos a su vez, inciden en el mantenimiento y preservación paisajística. A su vez, el estado de conservación de los recursos tiene relación directa con el uso eficiente y sostenible de los mismos. Esta relación circular que se establece entre recursos $\leftrightarrow$ coberturas $\leftrightarrow$ usos $\leftrightarrow$ paisajes se ve alterada con frecuencia por procesos que necesariamente derivan en una alteración de los dos extremos, los valores naturales y paisajísticos del medio. Las tipologías de usos y su nivel de explotación influyen en las diferentes unidades del paisaje, y la transformación de estas unidades puede dar lugar a cambios en los usos del suelo (Hietel et al., 2004). Por lo tanto, procesos, funciones y estructuras del paisaje guardan una relación muy estrecha con la dominante de los usos del suelo en un territorio dado. El entendimiento de estas dinámicas debe ser de utilidad en el análisis de cambios en las coberturas y usos del suelo y predecir comportamientos futuros.

Este complejo entramado de relaciones refuerza la componente natural y paisajística en los procesos de naturbanización, planteamientos que han sido centrales en las investigaciones más recientes. Los resultados reflejan la importancia de los análisis comparativos al tiempo que invitan a su abordaje desde una visión internacional (Prados, 2009). De una parte en Barcelona se han desarrollado investigaciones sobre la dualidad de las áreas de montaña y la delimitación de los tipos de paisaje en el parque nacional de Aigüestortes-Sant Maurici y los parques naturales de Cadí-Moixeró y Alt Pirineu (Prados \& Tulla, 2010). En Portugal, la Escuela de Ingeniería Civil de la Universidad de Minho ha analizado la expansión del fenómeno urbano en el área de influencia del parque nacional de Peneda-Gêres. Lo que ha permitido indagar sobre procesos asociados de abandono y marginalidad del espacio agrario, y sobre el análisis de la estructura ecológica del paisaje en territorios de baja densidad (Fernández, Prados \& Lourenço, 2010).

Si la naturbanización implica que los valores ambientales y los paisajes culturales del territorio son claramente apreciados por la población, el marco territorial y el paisaje deben ocupar un lugar preferente. Los resultados de los procesos de naturbanización investigados confrontan el aumento de la presión sobre el espacio construido y la intensificación agrícola, con situaciones de marginalidad y abandono de modelos productivos extensivos.

\section{METODOLOGIA Y RESULTADOS}

Las metodologías de análisis y evaluación de cambios en las coberturas y usos del suelo y sus relaciones con el medio natural y los paisajes están bien asentadas en Geografía y Ecología del Paisaje. El punto de partida puede emplazarse en el trabajo del geógrafo alemán C. Troll, reconocido por su aportación temprana al funcionamiento del paisaje como expresión combinada de las interacciones entre medio natural y cultural (Troll C., 1950). R. Forman ha reconocido las aportaciones de Troll en su concepción de la moderna la Ecología del Paisaje por su componente territorial (Forman \& Godron, 1986). Su concepción del paisaje como un tapiz formado por piezas que se conectan entre sí por medio de corredores tiene un claro trasfondo geográfico (pacht-corridor-matrix). Igualmente merece destacarse la labor del profesor Zonnelveld (1995) en la articulación de una metodología para la evaluación de suelos (land evaluation). Finalmente, los trabajos de 
R. Bunce sobre paisajes y aprovechamientos agrarios, o los de Turner y sus metodologías para la evaluación de cambios en las coberturas y usos del suelo merecen ser destacados por su contribución metodológica y aplicada (Turner \& Gardner, 1991; Bunce et al., 1993).

En todos estos trabajos y en paralelo a la evolución tecnológica, la información básica para el análisis de cambios en coberturas y usos muestra una estrecha dependencia de los avances en la recogida de imágenes teledetectadas. Las fotografías aéreas dieron paso a imágenes de satélite, que más tarde han dado lugar a otros productos más elaborados como las ortofotos y/o las imágenes de satélites de alta resolución. La consolidación de estas herramientas como suministradoras de datos geográficos y el desarrollo de programas informáticos para su tratamiento y análisis han propiciado el desarrollo de metodologías y aplicaciones bien diversas. Resultados palpables se encuentran también en las cartografías de coberturas y usos, ya se trata de series nacionales o regionales como de trabajos más específicos para áreas concretas de especial interés (Feria et al., 2002; Salvà et al., 2004); o del estudio comparado a partir de cartografías catastrales y mapas de coberturas y usos del suelo (Badia \& Rodríguez, 2005).

EL periodo temporal de creación del parques Nacional de Sierra Nevada, el primero a ser trabajado en la Península Ibérica es importante para seleccionar la cartografia con referente a las datas de su producción. En España esta data coincide con dos fuentes cartográficas digitales de gran importancia para la evolución de los usos del suelo: el Mapa de Cultivos y Aprovechamientos de 1975 y el Mapa de Cultivos Agrícolas de 1999 (Ministerio de Agricultura, Pesca y Alimentación, 1975; Consejería de Agricultura y Pesca, 1999). Ambas fuentes marcan respectivamente, el inicio de procesos de intensificación sobre áreas que acusaban ya cierta marginalidad; y la combinación de procesos de abandono agrario y especialización asociados a la entrada en juego de políticas de preservación natural y desarrollo turístico. La acción conjunta de estas fuerzas divergentes confiere una importancia extraordinaria a la evolución de los usos del suelo en este periodo. Ambas fuentes conforman también, proyectos de sistemas de información geográfica de ámbito estatal y regional haciendo posible la actualización de la información cartográfica y estadística de primer interés. Con ello se persigue un doble objetivo: validar la aportación de ambas cartografías como documentos clave en el estado de los usos del suelo en la comunidad andaluza; y establecer instrumentos de homogeneización de las leyendas que permitan su empleo comparado en diferentes ámbitos (Fernandez y Prados, 2010).

Así, la metodología para el análisis de la cartografía parte de un conocimiento profundo de cuál ha sido la evolución histórica del área de estudio, y en particular, de los cambios en los usos del suelo. De acuerdo con ello, la metodología aplicada desarrolla las fases recogidas en la figura 1:

1. Preparación de la información digital.

Selección y unión de las hojas a escala 1:50.000 en los años disponibles en España (1975 y 1999). En total se seleccionan ocho hojas, de las que se visualiza el campo correspondiente a los usos del suelo.

2. Análisis preliminar de las coberturas temáticas.

La relación de categorías en la leyenda de cada producto cartográfico ha de ser analizada con detenimiento, como paso previo a la simplificación de las leyendas adecuándolas a aquellas presentes en el ámbito de estudio; la homogeneización de categorías; y su 
agrupación y jerarquización. En el caso del parque nacional de Sierra Nevada las categorías presentes en el área de estudio han sido de un total de 19 en 1975 y 24 en la de 1999.

3. Homogenización de la leyenda.

Para poder realizar un análisis comparado de los usos del suelo es fundamental trabajar con una leyenda común. Se establecieron tres agrupaciones, según fuesen categorías coincidentes, equivalentes y no coincidentes. De ello resulta la leyenda final organizada en 14 categorías en las que se basa el análisis comparado de los usos del suelo en el periodo 1975/99 en la cuenca del Guadalfeo.

4. Generación de un Sistema de Información Geográfica.

Esta última fase corresponde a la generación cartográfica y estadística de la información relativa a cambios en los usos del suelo, una vez se ha obtenido la leyenda común para las coberturas de ambos mapas. La estimación de las superficies ocupadas por polígonos asignados a cada una de las tipologías de usos establecidas en las categorías irá seguida de un análisis de la dirección de los cambios detectados. La herramienta ArcGis 9.2. ha sido empleada, junto con la base de datos asociada Access, en el tratamiento de los datos correspondientes a la base de datos espacial asociada a la cartografía digital. Las categorías son analizadas de forma tanto cualitativa como cuantitativa mediante consulta SQL (Structured Query Language) para la estimación de los cambios en los usos del suelo, su dirección y superficie. El resultado final es la generación de cartografías adaptadas al ámbito de estudio para 1975 y 1991; y una segunda cartografía de permanencias y cambios en los usos del suelo en el periodo 1975/1999.

Esta metodología ha sido seguida para varios parques nacional incluso en Portugal para el parque nacional de Peneda-Gerês (véase Figura 1). Esto permite hacer una evakuación más rápida de los cambios.
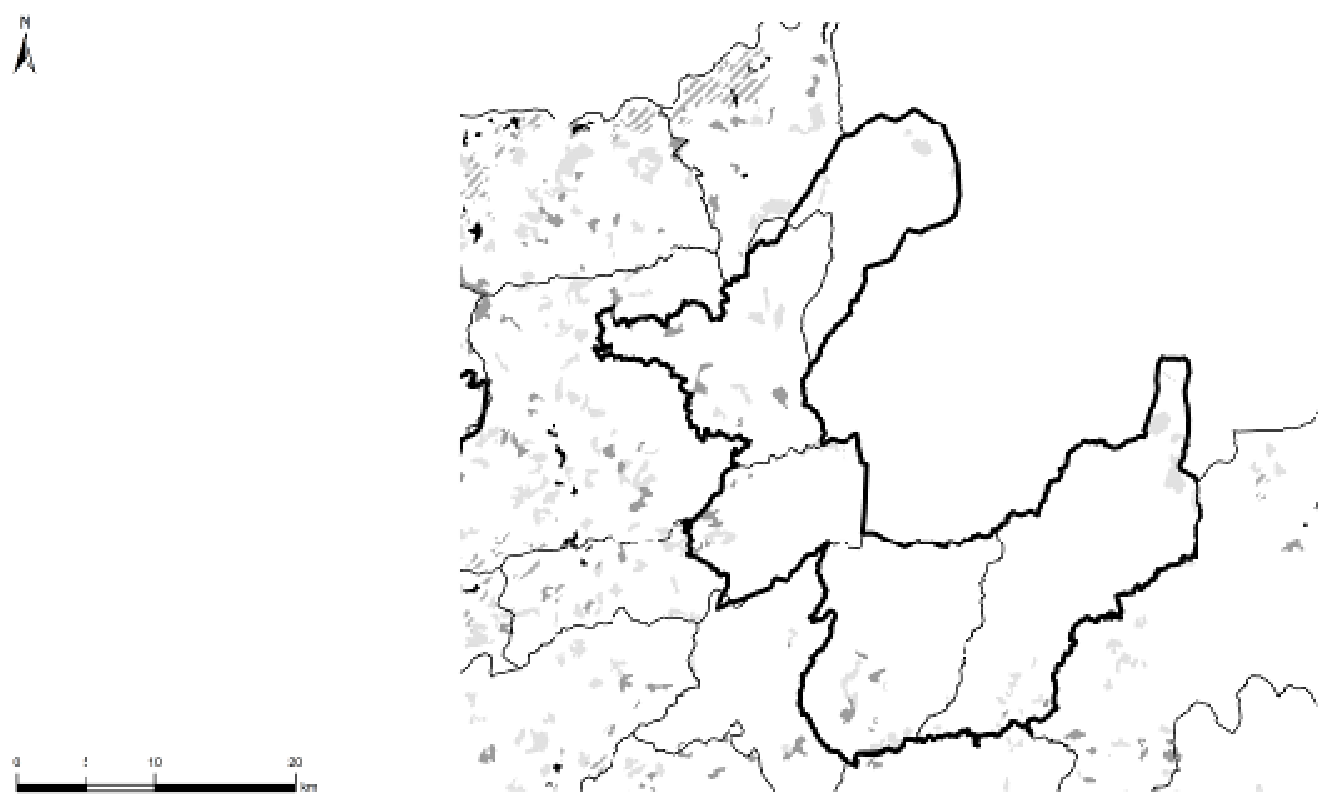

Fuente: (Lourenço, Quental y Barros, 2008).

Fig 1: Mapa con las alteraciones de los usos del suelo en parque nacional de Peneda-Gerês y su entorno. 
La metodología adoptada por Prados (2004) fue utilizada para otros territorios de España y también para Francia y Portugal.

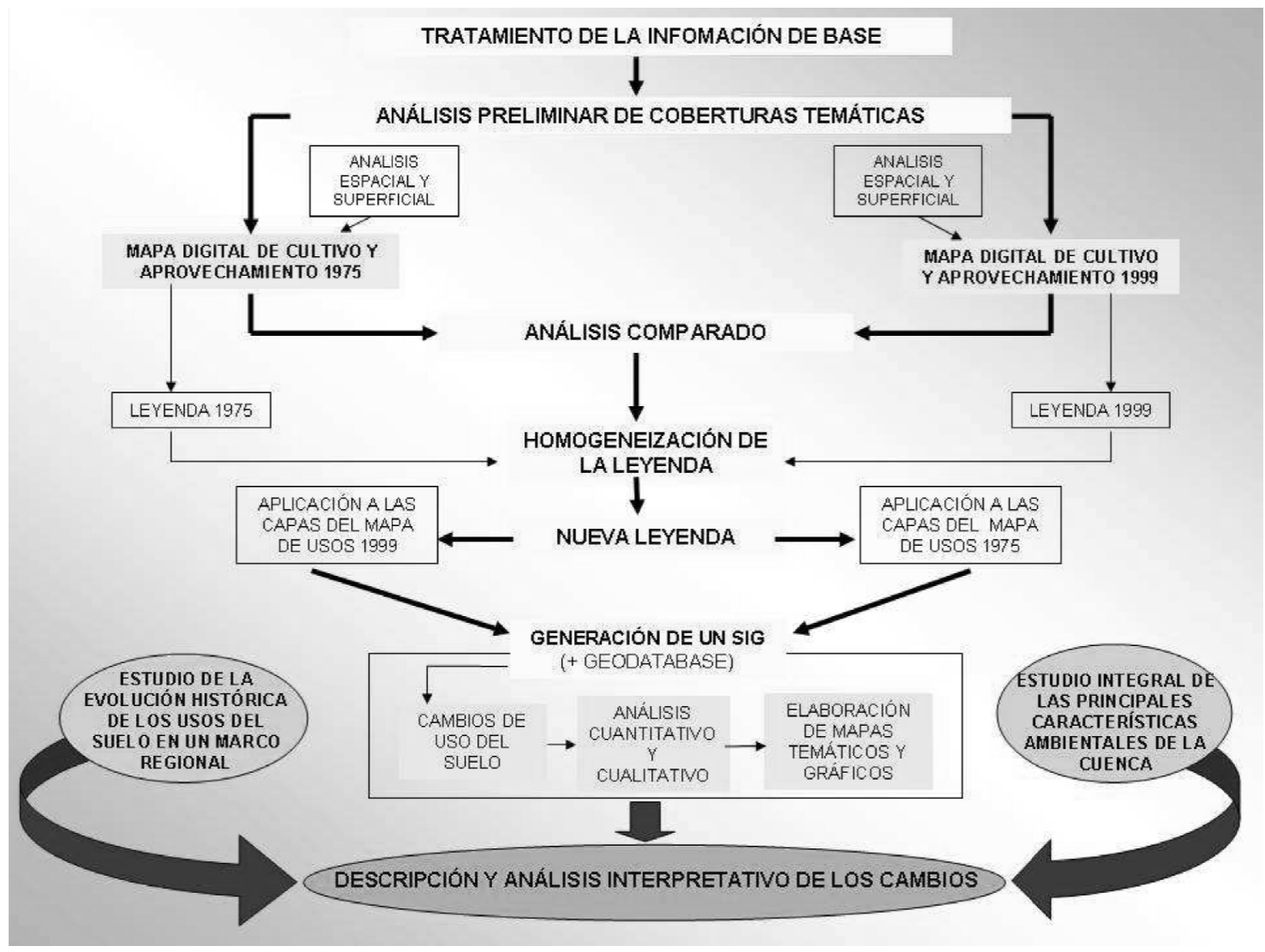

Fig 2: Síntesis del Esquema Metodológico

Los estudios ya desarrollados indician la posibilidad de la existencia de naturbanización en algunos territorios europeos.

\section{CONCLUSION}

El análisis de los cambios en los usos del suelo y su trascendencia en la naturbanización se analiza en este artículo en relación con las pesquisas desarrolladas para los parques nacionales de Sierra Nevada en España y de Peneda Gêres en Portugal. Se han investigado los desarrollos urbanos en los usos intensivos, y los cambios en los usos extensivos de forma genérica. Estos dos ámbitos son ejemplos claros de la relación entre medio natural y medio rural. Es por ello que el análisis temporal de los cambios en los usos del suelo se circunscribe a una etapa decisiva en la conformación actual de estos territorios. En el último cuarto del siglo XX culmina el proceso emigratorio comenzando una etapa de estabilidad en el crecimiento con signos de recuperación demográfica. Es también un periodo de modernización y especialización agraria que afecta tanto a la agricultura comercial como a las explotaciones familiares: la integración española y portuguesa en la Comunidad Europea y todo el corpus normativo en materia de desarrollo rural tiene asimismo gran incidencia en este ámbito. En este contexto, la creación de los parques nacionales de Sierra Nevada y de Peneda Gêres les confiere un reconocimiento explícito a su alto valor natural y paisajístico.

Se demuestra cómo estos procesos se asocian al abandono de actividades extensivas 
tradicionales y al desarrollo de procesos intensivos muy focalizados espacialmente, lo que sin duda genera un impacto en los ecosistemas. Mientras que la mayor parte de los trabajos sobre movimientos de población y reestructuración de las áreas rurales analizan el hecho en dependencia del comportamiento de las áreas urbanas, la hipótesis de la "naturbanización" valora la importancia del reconocimiento ambiental como justificación de las nuevas pautas en la movilidad y preferencias residenciales de la población. El concepto se ubica en este contexto en el que cobra importancia la caracterización sociodemográfica de las áreas naturbanizadas, intentando identificar la existencia de procesos de movilidad y el perfil de los nuevos residentes en relación con la conservación ambiental y paisajística. El otro eje sobre el que se sustenta la hipótesis guarda relación con las actividades económicas que están siendo reactivadas o las nuevas que surgen utilizando la presencia del parque natural. La naturbanización contribuye a identificar el valor mercantil de los parques, en relación tanto a las actividades económicas que se apoyan en su existencia como a la inversión pública en materia medioambiental. La hipótesis sobre la existencia de los procesos de naturbanización ha sido testada en relación con los cambios demográficos en las áreas de influencia de parques nacionales europeos. Los resultados iniciales de las investigaciones confirman la existencia de estos cambios demográficos en el conjunto de la red de parques nacionales en España y Portugal, y constatan la importancia de la calidad del entorno residencial, la accesibilidad y la posibilidad de desarrollar actividades económicas como factores de atracción de población hacia estos espacios. El desbordamiento de las zonas urbanas se acompaña de la invasión de áreas agrícolas y de alto valor natural, siendo de fuerte intensidad en casos muy señalados.

\section{REFERENCIAS}

Badia M. y Rodríguez F. (2005) Una nueva metodología para la recontrucción de la evolución histórica del paisaje agrario, Geofocus, Revista Internacional de Ciencia y Tecnología de la información Geográfica, 68-78.

Berry, B. J. (1976) Urbanization and Counterurbanization, Nueva York: Arnold.

Berry, B. J. (1978) The Counterurbanisation Process: How General?, N. Hansen (Ed.) Human Settlement Systems, 25-50 Cambridge: Ballinger.

Bills, N. \& Gross, D. (2005) Sustaining multifunctional agricultural landscapes: comparing stakeholder perspectives in New York (US) and England (UK), Land Use Policy, vol. 224, pp 313-321.

Brown D.L. y Wardell J.M. (eds.) (1980) New Directions in Urban-Rural Migration. The Population Turnaround in Rural América, Nueva York: Academic Press.

Bunce, R. G. H., et al. (1993) Landscape Ecology and Agroecosystems, Lewis publ., Boca Raton, Florida, 336 pp.

Camarero, L. A. (1993) Del éxodo rural y del éxodo urbano, Madrid: Ministerio de Agricultura, Pesca y Alimentación. 
Cózar Valero, M. ${ }^{a}$ E. (2000) Los recursos humanos en un espacio natural protegido:Sierra Nevada, Cuadernos Geográficos, 30: 325-346.

Champion, A. (1989), Counterurbanization: The change peace and nature of population deconcentration, Londres, UK: Edward Arnold.

Delgado, C. (2008), Urbanización sin fronteras. El acoso urbanístico a los espacios naturales protegidos, Boletín de la Asociación de Geógrafos Españoles, no 47, pp. 271 310.

Elbersen, B. (2005) Combining Nature Conservation and Residential Development in the Netherlands, England and Spain", Journal of Environmental Planning and Management 48: 37-63.

Elbersen, B. y Huigen P. (1998) The presence of protected nature and regional development: residencial development around the nature area of Doñana, I. Bowler, C. Bryant \& P. Huigen (eds.) Dimensions of sustainable Rural Systems, Utecht: Netherlands Geograhical Studies, 181-191.

Elbersen, B. y Prados, M. J. (1999) Desarrollo rural y calidad de vida en el entorno del Parque Nacional de Doñana, Estudios Regionales n ${ }^{\circ}$ 55: 47-76.

Esteban, A. (coord.) (2003) La humanización de las altas cuencas del Garona y las Nogueras, Madrid: Ministerio de Medio Ambiente.

Feria, J. M. (1998), El papel de las migraciones en la evolución de la población andaluza. Tendencias recientes y perspectivas futuras, Boletín Económico de Andalucía, 24, 109118.

Feria, J. M., et al. (2002) Redes de centros históricos en Andalucia, Seville: Regional Government of Andalusia

Fernández M., Prados M. J. \& Lourenço, J. M. (2010) Naturbanization and environmental conservation in a climate change context: the case of PNPG, International Workshop The History of Environment and Global Climate Change, Braga, in press.

Fielding, A. J. (1986) Counterurbanization, M. Paccione (ed.) Population Geography: progress and prospects, Londres: Croom Helm, 224-256.

Fleskens L., Duarte F. \& Eicher, I. (2009) A conceptual framework for the assessment of multiple functions of agro-ecosystems: A case study of Trás-os-Montes olive groves, Journal of Rural Studies, vol. 25-1, pp 141-155.

Fontaine, C. M. \& Rounsevell, M. D. A. (2009) An agent-based approach to model future residential pressure on a regional landscape, Landscape Ecology, vol. 24-9, pp 1237-1254.

Forman R. T. T. y Godron, M. (1986) Landscape Ecology, John Wiley and Sons, New York, NY. 619pp. 
García-Bellido A. y García J. (2002) La cuestión rural: patología urbanística del espacio rústico, Ciudad y Territorio: Estudios Territoriales, 132: 277-323.

Gómez, A. (2007) Componentes del valor del paisaje mediterráneo y el flujo de servicios de los ecosistemas, Ecosistemas, vol. 16-3, pp. 97-108

Hall, C. (2008) The landscape aesthetics of functional change in agriculture: how do they impact on rural residents in Scotland?, 8th International Farming Systems Association Symposium. Clermont Ferrand: International Farming System Association, pp. 1-10.

Hietel, E., et al. (2004) Analysing land-cover changes in relation to environmental diversity in Hesse, Germany, Landsc. Ecol. 19.

McDonald, R. I. et al. (2009) Urban effects, distance and protected areas in an urbanizing world, Landscape and Urban Planning, vol. 93-1, pp 63-75.

Lourenço, J. M.; Quental, N.; Barros, F. (2008) Naturbanization and sustainability at Peneda-Gerês National Park, in Naturbanization: New identities and processes for rural-natural areas, Taylor \& Francis Group, pp. 45-73.

Prados, M. J. (2000) La revitalización de espacio rural andaluz en el contexto de las políticas de protección ambiental, F. García, A. Larrull y R. Majoral (Eds.), Los Espacios Rurales en el Cambio de Siglo: Incertidumbres ante los procesos de globalización y desarrollo, Lleida: Universidad de Lleida, 9 pags..

Prados M. J. y Cunningham, C. (2002) Calidad ambiental y nuevas pautas en la movilidad residencial de la población. Propuesta metodológica para el estudio de procesos de naturbanización, Los Espacios Rurales entre el hoy y el mañana, Santander: Universidad de Cantabria, 425-433.

Prados, M. J. (2005) Territorial recognition and the control of changes and dynamic rural areas. Analysis of naturbanisation process in Andalucía-Spain, Journal of Environmental Management and Planning- special issue vol. 48, pp. 65-83.

Prados, M. J. (2009) Naturbanization. New identities and processes for rural-natural areas, Londres: Taylor \& Francis Group.

Prados, M. J. y Tulla, A. F. (2010) New rural landscapes alter naturbanization, Robinson G, Molinero F. \& Guerra (eds.) Procedings of III Anglo-Spanish Rural Geography Symposium, Canterbury: Rural Geography Research Group - Asociación de Geógrafos Españoles, pp. 109-126.

Salvà, M., et al. (2004) El análisis a gran escala y las nuevas tecnologias: una nueva interpretación de las superfícies de los tipos de vegetación y usos del suelo de Parque Natural de Montseny (Barcelona, Girona), Estudios en Biogeografia, Ed. Aster., Terrassa, 107-116. 
Swift, M. J., et al. (2004) Biodiversity and ecosystem services in agricultural landscapesare we asking the right questions?, Agriculture, Ecosystems \& Environment, vol. 104-1, pp. 113-134.

Troll, C. (1950) Die geographische Landschaft und Ihre Erforschung, Stadium Generale 3(4/5): 163-181.

Tulla, A. (1993) Procés de transformació agrària en àrees de muntanya, Barcelona: Insttut Cartogràfic de Ctalunya.

Turner, M. G. \& Gardner, R. H. (1991) Landscape Ecology in Theory and Practice: Pattern and Process, Springer, USA.

Williams, A.S. \& Jobes, P.C. (1990) Economic and quality-of-life considerations in urbanrural migration, Journal of Rural Studies, $6 \mathrm{n}^{\circ} 12,187-194$.

Zonneveld, I. S. (1995) Land Ecology, SPB Academic Publishing bv, Amsterdam, 199 pp. 